\title{
Field-regulated switching of the magnetization of Co-porphyrin on graphene
}

\author{
D. Klar, ${ }^{1, *}$ S. Bhandary, ${ }^{2}$ A. Candini, ${ }^{3}$ L. Joly, ${ }^{4}$ P. Ohresser, ${ }^{5}$ S. Klyatskaya, ${ }^{6}$ M. Schleberger, ${ }^{1}$ M. Ruben,,${ }^{4,6}$ M. Affronte, ${ }^{3,7}$ \\ O. Eriksson, ${ }^{2}$ B. Sanyal, ${ }^{2, \dagger}$ and H. Wende ${ }^{1}$ \\ ${ }^{1}$ Faculty of Physics and Center for Nanointegration Duisburg-Essen (CENIDE), University of Duisburg-Essen, \\ Lotharstraße 1, D-47048 Duisburg, Germany \\ ${ }^{2}$ Department of Physics and Astronomy, Uppsala University, Box 516, 75120 Uppsala, Sweden \\ ${ }^{3}$ Centro S3, Istituto Nanoscienze - CNR, via Campi 213/a, 41125 Modena, Italy \\ ${ }^{4}$ Universite de Strasbourg, Institut de Physique et de Chimie des Materiaux de Strasbourg, Campus de Cronenbourg, \\ 23 Rue du Loess, 67034 Strasbourg Cedex 2, France \\ ${ }^{5}$ Synchrotron SOLEIL, L'Orme des Merisiers, Saint-Aubin - BP 48, 91192 Gif-sur-Yvette, France \\ ${ }^{6}$ Institute of Nanotechnology, Karlsruhe Institute of Technology (KIT), D-76344 Eggenstein-Leopoldshafen, Germany \\ ${ }^{7}$ Dipartimento di Scienze Fisiche, Informatiche e Matematiche, Università di Modena e Reggio Emilia, via Campi 213/a, 41125 Modena, Italy
}

(Received 8 November 2013; revised manuscript received 24 March 2014; published 14 April 2014)

\begin{abstract}
Different magnetic coupling mechanisms have been identified for a few monolayers of Co-porphyrin molecules deposited on a graphene-covered $\mathrm{Ni}(111)$ single crystal. A relatively strong antiferromagnetic coupling of the first molecular layer via graphene to the $\mathrm{Ni}$ crystal in comparison to a weaker intermolecular coupling gives rise to a complex field-dependent response of this hybrid system. By continuously increasing the magnetic field strength, the net magnetization of the molecular system switches from antiparallel to parallel to the field direction at 2.5 T. Utilizing x-ray absorption spectroscopy and x-ray magnetic circular dichroism, the element-specific magnetization and field dependence was probed. The nature of the magnetic couplings is identified by means of density functional theory and orbital-dependent susceptibilities.
\end{abstract}

The development of new materials for spintronic applications as an alternative to conventional devices is one of the major challenges of present research. Using the spin of organic materials may be a solution for the limitation of downscaling of current devices [1-3]. Organic molecules, such as metal porphyrins, provide convenient possibilities for a realization of such applications. In recent years, different groups have demonstrated that the magnetization of Mn- and Fe-porphyrins can be controlled by ferromagnetic coupling to ferromagnetic substrates [4-6]. Depending on the magnetization of the film, one can tune the magnetic moments of the molecules to lie either in plane or perpendicular to the surface. With an interlayer of oxygen, the coupling could also be changed, yielding an antiparallel alignment with respect to the magnetic moment of the substrate [7]. Compared to an interlayer of oxygen, graphene has strong advantages when it comes to tuning the exchange interaction between substrate and molecular magnet. The coupling energy via oxygen to the first molecular layer is very high $[7,8]$ compared to using graphene, as presented in this paper. A too strong coupling between the magnetic moments of the molecule and the substrate prevents the possibility to tune the composite system's net magnetization with, e.g., an external magnetic field. In the present work, we are indeed able to switch the net moment of the Co-octaethylporphyrin (OEP) molecules adsorbed on graphene reversibly between parallel and antiparallel orientations with a magnetic field. The antiferromagnetic coupling on a graphene-covered $\mathrm{Ni}(111)$ was calculated for other molecules [9]. Hermanns et al. recently detected an antiferromagnetic coupling of a CoOEP submonolayer to a graphene-covered Ni film [10,11], which

\footnotetext{
*david.klar@uni-due.de

†Biplab.Sanyal@physics.uu.se
}

followed a study of CoOEP/Ni(100) [12]. Since its discovery, graphene [13] has become one of the most important topics in various fields of material science. Its unique electronic properties make it invaluable for different applications [14]. For instance, by density functional theory (DFT) calculations, graphene was shown to be a reversible spin manipulator for Fe-porphyrin molecules [15].

In this paper, we demonstrate the field dependence of the net magnetization of three layers of CoOEP molecules and a graphene-covered $\mathrm{Ni}(111)$ single crystal. Via elementspecific x-ray magnetic circular dichroism (XMCD), we can individually determine the magnetization of the $\mathrm{Ni}$ crystal and the molecules. The net magnetization of the Co-porphyrin molecules is the sum of two different contributions, i.e., a constant antiparallel magnetization from the first layer and a field-dependent contribution from the second and third ones. This behavior of the system enables us to switch the magnetization by regulating an external magnetic field. We are able to confirm the antiferromagnetic coupling through the graphene layer with DFT calculations and compute the coupling energy as well as spatial- and orbital-dependent susceptibilities. The calculated field-dependent contributions to the magnetism from the two superposing signals fit the experimental data very well.

The samples were prepared in situ at a base pressure of $10^{-10}$ mbar. We produced a single-layer graphene on top of the clean $\mathrm{Ni}(111)$ single crystal in situ by heating the crystal in a propylene atmosphere, following Ref. [16]. Graphene is known to grow epitaxially on the $\mathrm{Ni}(111)$ surface [17]. Subsequently, the CoOEP molecules were thermally evaporated onto the graphene-covered $\mathrm{Ni}(111)$ crystal, while the substrate was held at room temperature. The thickness of three layers with an accuracy of $\pm 15 \%$ was controlled with a microbalance on top of the evaporator. All molecules are 

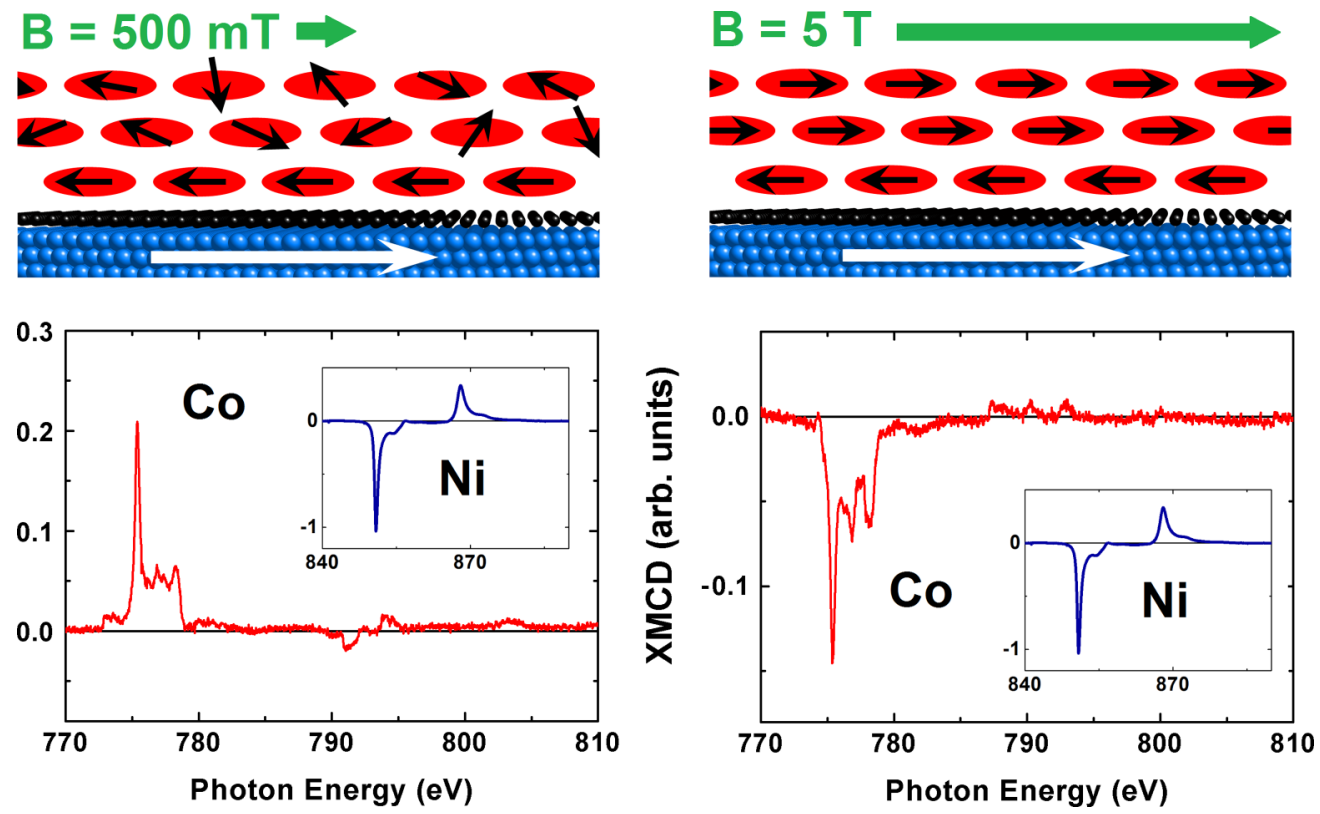

FIG. 1. (Color online) Top: Schematic images of the sample: $3 \mathrm{ML} \mathrm{CoOEP/graphene/Ni(111).} \mathrm{The} \mathrm{white} \mathrm{and} \mathrm{black} \mathrm{arrows} \mathrm{indicate} \mathrm{the}$ directions of the magnetic moments of $\mathrm{Ni}$ (blue) and $\mathrm{Co}$ (red) in a low magnetic field of $500 \mathrm{mT}$ (left) and in a high magnetic field of $5 \mathrm{~T}$ (right). Bottom: XMCD at the $\mathrm{Co}_{2,3}$ edges of the CoOEP molecules in the low magnetic field (left) and in the high magnetic field (right). Insets show the XMCD at the $\mathrm{L}_{2,3}$ edges of the saturated $\mathrm{Ni}$ crystal at $500 \mathrm{mT}$ and at $5 \mathrm{~T}$. All spectra are recorded at $T=2.8 \mathrm{~K}$ and $\Theta=70^{\circ}$.

oriented and lie parallel to the surface, as shown in Figs. 2 and 3 in the Supplemental Material [18]. The x-ray absorption spectroscopy (XAS) measurements in total electron yield were performed at the undulator beam line DEIMOS at SOLEIL. In the cryomagnet, we could reach a magnetic field up to $7 \mathrm{~T}$ and a temperature of $2 \mathrm{~K}$ at the sample holder. This results in a temperature of approximately $2.8 \mathrm{~K}$ on the sample surface. With the element-specific XMCD technique at the Co and $\mathrm{Ni} \mathrm{L}_{2,3}$ edges, we analyzed the magnetic field dependence of the net magnetization of the $\mathrm{Ni}(111)$ crystal and the CoOEP molecules.

We have performed first-principles density functional calculations with a full potential plane-wave-based code, VASP [19]. The unit cell used consisted of a slab of three $\mathrm{Ni}$ layers in the (111) direction having 108 atoms along with a graphene monolayer with 72 atoms $(6 \times 6$ supercell $)$ on top of the slab. A CoP molecule with 37 atoms was kept on graphene, yielding a total of 217 atoms in the simulation cell. It should be noted that the outermost chains of the CoOEP molecule have been replaced by $\mathrm{H}$ atoms in our simulations and, hence, we will denote the molecule as CoP. The size of the supercell was chosen in such a way that the magnetic centers of CoP are at least $15 \AA$ apart from each other in the lateral direction. A $3 \times 3 \times 1 k$-point mesh was chosen for geometry optimizations with a force tolerance of $0.01 \mathrm{eV} / \AA$. All of the atomic positions were relaxed except the ones in the lowest $\mathrm{Ni}$ layer, which is fixed to have the in-plane lattice constant as that of bulk Ni. A $4 \times 4 \times 2 k$-point mesh was considered for calculating total energies and other properties. We have employed a DFT $+U$ approach within the Hubbard model [20] to incorporate strong Coulomb interaction with the Coulomb parameter $U$ and exchange parameter $J$ fixed as 4 and $1 \mathrm{eV}$, respectively, for Co $d$ orbitals. These parameters have been shown [21] to reproduce certain experimental results for $3 d$ transition-metal-centered porphyrin molecules. All of the calculations were performed including an empirical form of dispersion correction given by Grimme [22].

Figure 1 shows the XMCD spectra at the $\mathrm{L}_{2,3}$ edges for the CoOEP molecules as well as for the $\mathrm{Ni}$ single crystal, along with the schematic illustrations of the corresponding samples. The black arrows illustrate the magnetic moments of each Co ion, while the white arrow represents the macroscopic magnetization of the $\mathrm{Ni}$ crystal. The spectra were recorded at a temperature of $2.8 \mathrm{~K}$ and at grazing $\mathrm{x}$-ray incidence $\left(\Theta=70^{\circ}\right.$ with respect to the surface normal), with the external magnetic field parallel to the beam. The XMCD signal at the $\mathrm{Ni} \mathrm{L}_{2,3}$ is plotted as the inset in both Co spectra, showing the same XMCD signal for both magnetic fields, i.e., $500 \mathrm{mT}$ and $5 \mathrm{~T}$, because the magnetization of the crystal is saturated for $B>200 \mathrm{mT}$. In the spectrum at the high magnetic field of $B=5 \mathrm{~T}$, the XMCD intensity at the $\mathrm{Co}_{3}$ edge is negative, just like the $\mathrm{L}_{3}$ edge of the Ni XMCD. However, in a lower magnetic field of $500 \mathrm{mT}$, the XMCD signal at the $\mathrm{Co} \mathrm{L}_{3}$ edge changes its sign, whereas the shape of the XMCD signal is unchanged. Further details about the field-dependent XMCD are presented in Fig. 4 in the Supplemental Material [18]. At a temperature of $2.8 \mathrm{~K}$, the spin fluctuation of the weakly coupled, paramagnetic Co-porphyrin molecules is too strong to determine a net alignment of the Co moments in a small magnetic field of $500 \mathrm{mT}$. Hence, the XMCD signal for low magnetic fields is dominated by the first CoOEP layer which is coupled antiferromagnetically to the ferromagnetic substrate. All of the magnetic moments of the Co ions in the first layer are oriented antiparallel to the magnetization of the Ni film and therefore antiparallel to the applied field. The second and third layers are only weakly coupled (see 


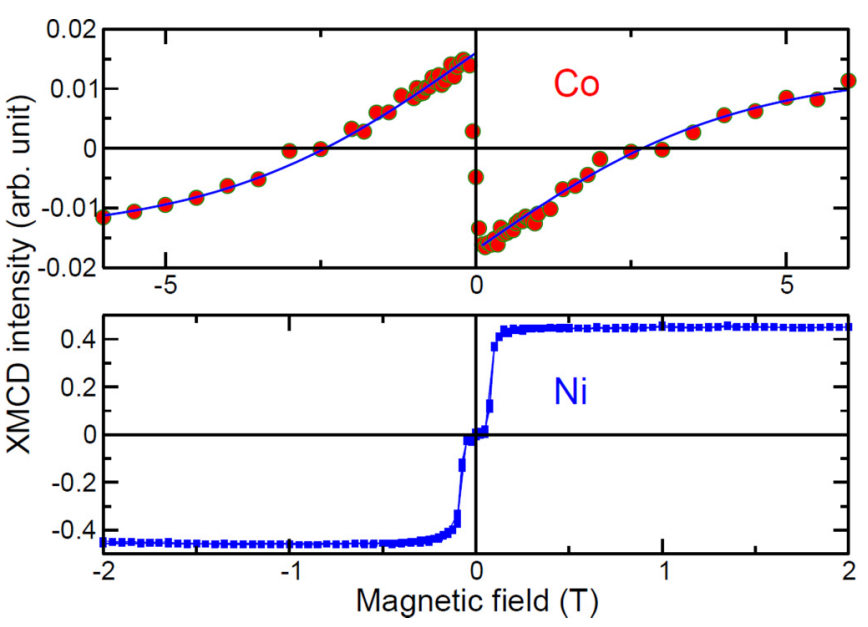

FIG. 2. (Color online) Magnetization curves between -2 and +2 $\mathrm{T}$ for $\mathrm{Ni}$ (blue) and between -6 and $+6 \mathrm{~T}$ for $\mathrm{Co}$ (red), measured at $\Theta=70^{\circ}$ and $T=2.8 \mathrm{~K}$. The continuous line shows the fitted Co magnetization (see text for details).

details below) and are only slightly affected by the small field, as is expected for a paramagnetic system. These Co magnetic moments point in various directions and add up to a negligibly small magnetization in the field direction. In Fig. 2, this is demonstrated in a clear way where the magnetization curves at the $\mathrm{Co}_{3}$ and $\mathrm{Ni} \mathrm{L}_{3}$ edges are presented. In both cases, the measurements were performed at constant photon energies of $775.4 \mathrm{eV}$ for $\mathrm{Co}$ and $850.9 \mathrm{eV}$ for $\mathrm{Ni}$, at which the XMCD intensity is maximum, and normalized to pre-edge energies of 772.0 and $845.0 \mathrm{eV}$, respectively, while the magnetic field was varied. The magnetization of the $\mathrm{Ni}$ single crystal increases strongly in a small magnetic field and saturates at about $200 \mathrm{mT}$. Simultaneously, at this field strength, the Co magnetization rises to its maximum value, antiparallel to the field, demonstrating the antiferromagnetic coupling. In higher fields, the $\mathrm{Ni}$ magnetization remains constant, while the absolute value of the Co XMCD signal decreases and reaches zero at a field of nearly $2.5 \mathrm{~T}$. At this point, the magnetization of the molecules in the second and third layers annihilates the magnetization of the first one. In higher fields, the net magnetization switches its sign because the amount of parallel aligned magnetic moments is higher than the antiparallel magnetic moments of the first layer. Close to zero magnetic field, the magnetization of the $\mathrm{Ni}$ crystal vanishes due to spontaneous formation of magnetic domains and so does the magnetization of the Co ions. The hysteresis curve for Co, as shown in Fig. 2, has been fitted with the superposition of two contributions, $M(B)=M_{1} \tanh \left[\mu_{B}(B-\right.$ $\left.\left.B_{e x}\right) / k_{B} T\right]+M_{2,3} \tanh \left(\mu_{B} B / k_{B} T\right)$, with $S=\frac{1}{2}$ and $B_{e x}=$ $72 \mathrm{~T}$, i.e., the exchange field between the Co moment of the first molecular layer and the $\mathrm{Ni}$ surface, obtained from our DFT $+U$ calculations. The fitting parameters $M_{1}$ and $M_{2,3}$ represent the saturation magnetizations of the first layer, and the second and third layers, respectively. Since the exchange field is much larger than the external magnetic field, $\tanh \left[\left(B-B_{e x}\right) / k_{B} T\right]$ is approximately -1 , leading to a field-independent antiparallel contribution $-M_{1}$ originating from the first layer. We fit the second and third layers' contribution paramagnetically because of the weakness of the intermolecular interaction. The fit yields $M_{2,3}=$ $1.8\left|M_{1}\right|$, indicating that the film thickness is approximately 2.8 ML, which is in good agreement with our assumption of $3 \mathrm{ML}$.

Our DFT $+U$ calculations also reveal an antiferromagnetic coupling between the Co moment in $\mathrm{CoP}$ and the Ni surface. However, the coupling strength is much smaller compared to chemically adsorbed molecules on magnetic surfaces [23]. Due to the presence of the graphene layer, $\mathrm{CoP}$ is physisorbed which significantly weakens the magnetic coupling. The chemical properties of graphene are also modified due to strong hybridization with the $\mathrm{Ni}(111)$ layer. A perfect lattice matching helps one of the $\mathrm{C}\left(\mathrm{C}_{A}\right)$ sublattice atoms to chemically bind to $\mathrm{Ni}$ atoms, while the other $\mathrm{C}$ sublattice $\left(\mathrm{C}_{B}\right)$ atoms remain $s p^{2}$ bonded. This imbalance induces different magnetizations on different $\mathrm{C}$ sublattices. $\mathrm{C}_{A}$ develops a moment of $0.018 \mu_{B}$ and aligns antiparallel to the $\mathrm{Ni}$ moment, while $\mathrm{C}_{B}$ gets magnetized parallel to the Ni moment with a moment of $0.03 \mu_{B}$, yielding a ferrimagnetic graphene layer. We have, in the calculations, considered CoP adsorption sites on top of $\mathrm{C}_{A}, \mathrm{C}_{B}$, and in the middle of the graphene hexagon, which we refer to in the rest of this paper as Top-A, Top-B, and Hexagon, respectively. We find from our calculations that the physisorbed CoP finds itself spatially $3.07,3.04$, and $3.11 \AA$ away from the graphene layer for the Top-A, Top-B, and Hexagon sites, respectively. The Top-A site is found to be the most stable one, while the Top-B and Hexagon sites are 14.6 and $23.5 \mathrm{meV}$ higher in energy.

From the total-energy calculations, we find that the central Co atom in CoP couples antiferromagnetically with the underlying Ni surface for all adsorption sites, in agreement with our experiments. In contrast to a previous study [10] for a similar system, we find that the exchange-coupling energies $\left(E_{\mathrm{FM}}-E_{\mathrm{AFM}}\right.$, where $E_{\mathrm{FM}}$ and $E_{\mathrm{AFM}}$ represent the total energies in the ferromagnetic and the antiferromagnetic orientation, respectively) are small $(4.2,9.9$, and $3.1 \mathrm{meV} /$ cell for Top-A, Top-B, and Hexagonal adsorption sites, respectively). While oxygen as a buffer layer has a saturation coverage at half a monolayer, e.g., on $\mathrm{Co} / \mathrm{Cu}(100)[7,8]$, leading to well separated and localized $\mathrm{O} p_{z}$ orbitals, the $\mathrm{C} \pi$ orbitals build a delocalized $\pi$ band. Due to this delocalization, the molecules couple antiparallel, independent of their absorption site. We want to comment that if $\mathrm{CoP}$ is replaced by FeP, studied in Ref. [24], Fe couples ferromagnetically to Ni for the Top-A site due to the involvement of different types of orbitals in mediating the exchange interaction.

To understand the exchange interactions, we present, in Fig. 3, the density of states of the out-of-plane orbitals for (CoP)-Co, (CoP)-C, (CoP)-N, (Graphene)-C, and surface layer $\mathrm{Ni}$ for the most stable configuration, Top-A. The exchange splittings of the Co and Ni states demonstrate the antiferromagnetic configuration, which is further vividly represented in the magnetization density plot (inset). The magnetization density (blue) that appears on the Co atom is solely from a singly occupied $d_{z^{2}}$ orbital and couples antiferromagnetically with Ni moments (red). It may be observed that the Ni $3 d$ and graphene $p_{z}$ states hybridize and should therefore have a stronger chemical interaction than between graphene and 


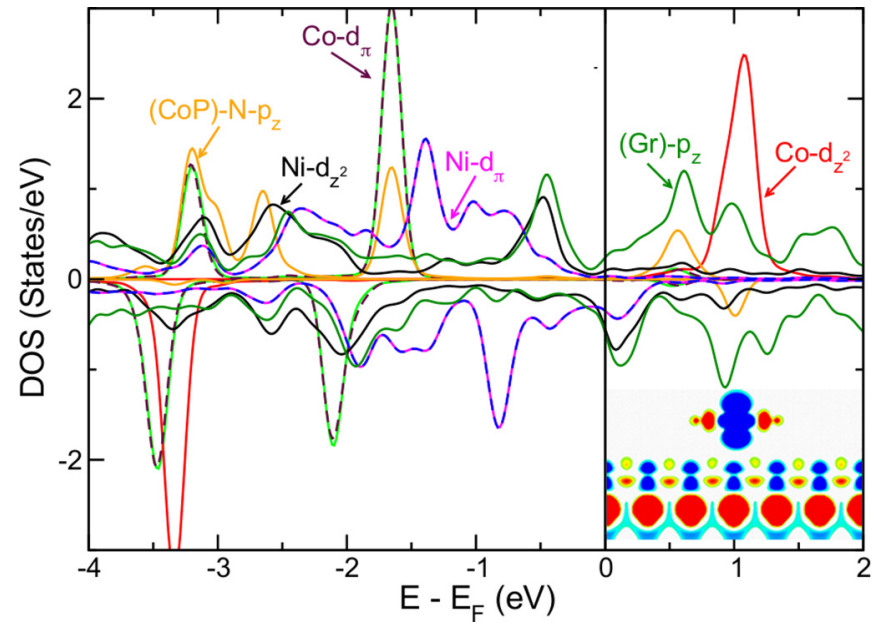

FIG. 3. (Color online) Out-of-plane contribution to the DOS, resolved on $\mathrm{C}, \mathrm{N}$, and $\mathrm{Co}$ states projected onto the Co-porphyrin molecule, labeled (CoP)-Co, (CoP)-C, (CoP)-N. We also show a projection of graphene $\mathrm{C}$ atoms, labeled (Graphene)-C atoms. Projections are done on specific $m_{l}$ projected orbitals. Inset: Magnetization density cross section, which depicts antiferromagnetic coupling between $\mathrm{Ni}$ and $\mathrm{Co}$ along with graphene sublattice magnetization. Red and blue colors indicate positive and negative densities on $\mathrm{CoP}$ (on top), graphene (middle layer), and the first Ni layer of our unit cell.

CoP. Also, there is no hybridization visible between $\mathrm{Ni}$ and Co $3 d$ orbitals, which excludes a direct exchange mechanism between $\mathrm{CoP}$ and $\mathrm{Ni}$ substrate. Instead, exchange is indirect and is mediated via the graphene states.

To shed more light on the exchange coupling between surface $\mathrm{Ni}$ and $\mathrm{Co}$ atoms, we have analyzed the orbital dependence of the susceptibility, which is proportional to the interatomic pair-exchange interaction (considering energyindependent local potentials), expressed as $\chi_{m m^{\prime}}^{i j}\left(E_{F}\right) \propto$ $\operatorname{Im} \int^{E_{F}} d E \sum_{m^{\prime \prime}} G_{m m^{\prime \prime}}^{i j \uparrow}(E) G_{m^{\prime \prime} m^{\prime}}^{j i, \downarrow}(E)$, with $m, m^{\prime}$ being magnetic quantum numbers $\left(m_{l}\right)$ for $d$ orbitals, while $G^{i j, \sigma}$ denotes the Green function between $i(\mathrm{Co})$ and $j$ (Ni atom beneath the $\mathrm{C}$ atom of the graphene layer in the Top-A position; see Fig. 3) atoms for the spin channel $\sigma(\sigma=\uparrow, \downarrow)$. The orbital-projected Green functions were generated by the projection of KohnSham orbitals onto spherical harmonics in a sphere around each atom. The $m_{l}$-dependent susceptibility as a function of Fermi energy $E_{F}$ is shown in Fig. 4. In these calculations, first $E_{F}$ was determined self-consistently (denoted as zero in Fig. 4). Then the value of $E_{F}$ was varied using a rigid band model by fixing the self-consistent electronic structure and calculating the orbital-dependent $\chi^{i j}$ for each $E_{F}$. It can be observed from Fig. 4 that the in-plane orbital contributions are almost zero throughout, while the out-of-plane $\left(d_{\pi}=\right.$ $d_{x z}+d_{y z}$ ) contribution is significantly below the Fermi energy. At $E_{F}=0$, the major contribution comes from the $d_{z^{2}}$ orbital with a relatively small contribution from $d_{x z / y z}$ orbitals. Note also that the contributions for $d_{z^{2}}$ and $d_{x z / y z}$ orbitals are of different sign, meaning that the total contribution (summed over orbitals) will be slightly reduced.

Now we discuss the exchange interactions between the molecular layers. As argued before, based on our experiments,

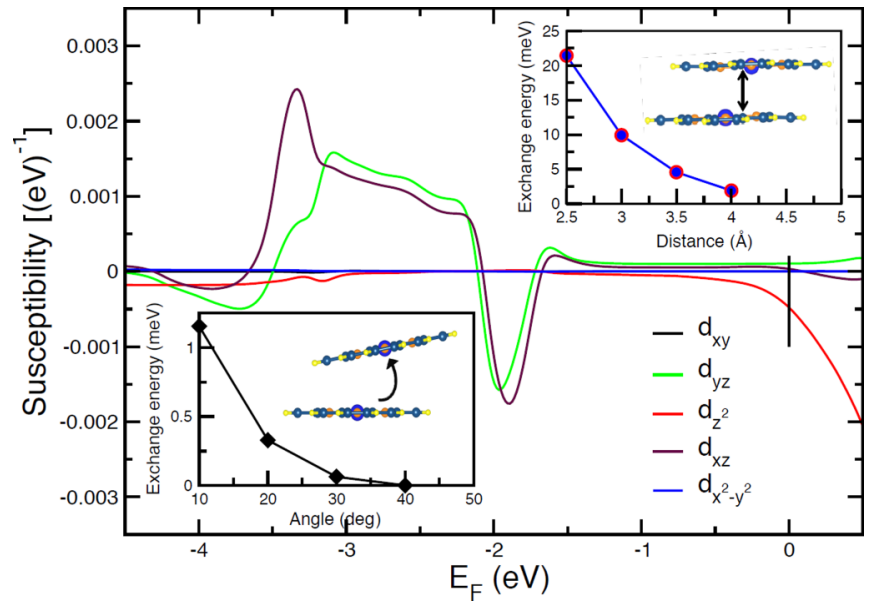

FIG. 4. (Color online) Orbital-dependent susceptibility as a function of Fermi energy. Note the dominance of the $d_{z}^{2}$ orbital at the Fermi energy $E_{F}=0$, relevant for our present system. Inset: Distance- and angle- (between two CoP molecules) dependent exchange energies. Schematic views of relative separation and rotation between two $\mathrm{CoP}$ molecules are given in the two insets.

the top and middle layers behave like paramagnets, while the lowest layer is exchange coupled to the Ni substrate via graphene. We have found from our calculations for a bilayer of $\mathrm{CoP}$ that the high-symmetric, perfectly parallel CoP layers are antiferromagnetically coupled to each other. The optimized geometry has the two Co atoms in two consecutive layers shifted laterally, which is also discussed in the literature for CoPc [25]. The spatial separation between the parallel layers reduces the exchange strength considerably due to lowering of orbital overlap, while randomness in the orientation has a higher impact in reducing interlayer exchange coupling. In Fig. 4 (inset), the sharp fall of exchange energy due to increasing separation between two CoP layers and angular deviations between them is shown. Two CoP molecules making an angle to each other not only increases the effective distance between Co atoms, but also causes the type of orbitals participating in the exchange to be modified. This results in a drastic decoupling of the exchange interaction between the layers. As mentioned before, this randomness in orientation can occur for dense molecular layers on surface due to the outermost ligands in CoOEP. The applied magnetic field thus can orient these loosely coupled layers and turn the whole system ferromagnetic, which confirms the argument of field-dependent switching of the magnetization. It should be noted that the out-of-plane magnetic anisotropy energy is high $(0.19 \mathrm{meV})$ compared to the extremely small in-plane anisotropy (easy-plane system) as given by our $\mathrm{DFT}+U$ calculations for $\mathrm{CoP}$ in the gas phase, in which we included spin-orbit coupling. This indicates that the field-dependent switching most probably takes place by in-plane rotation of Co moments.

In summary, we have demonstrated the switching of magnetization of three layers of CoP deposited on 1 ML graphenecovered Ni(111) substrates by external magnetic fields. From our analysis, with the aid of density functional calculations, we find that the $\mathrm{CoP}$ layer adjacent to the graphene layer is strongly exchange coupled antiferromagnetically to the $\mathrm{Ni}$ 
substrate, whereas the top and the middle CoP layers are coupled via very weak, short-ranged interactions and, hence, can easily be switched by external fields. This achievement is a result of an effective decoupling of the second and third molecular layers, generated by the graphene interlayer, which is the key to the field-regulated switching. The realization of a field-regulated switching of molecular magnets is an important step ahead towards future molecular spintronic concepts, as the spin injection into the organic layer can be regulated by a magnetic field.
We are grateful to Fadi Choueikani for assistance and to the SOLEIL staff for smoothly running the facility (Proposal No. 20120073). We also acknowledge support from the DFG (Grants No. SPP1459 and No. SCHL 384/15-1), the Swedish Research Council, Carl Tryggers Stiftelse, the KAW Foundation, eSSENCE, and the ERC (Project No. 247062 - ASD). The Swedish National Infrastructure for Computing (SNIC) is gratefully acknowledged for the allocation of supercomputing time. We acknowledge support from the Italian Ministry for Research (MIUR) through the FIR grant RBFR13YKWX.
[1] Z. H. Xiong, D. Wu, Z. V. Vardeny, and J. Shi, Nature (London) 427, 821 (2004).

[2] L. Bogani and W. Wernsdorfer, Nat. Mater. 7, 179 (2008).

[3] W. J. Cho, Y. Cho, S. K. Min, W. Y. Kim, and K. S. Kim, J. Am. Chem. Soc. 133, 9364 (2011).

[4] A. Scheybal, T. Ramsvik, R. Bertschinger, M. Putero, F. Nolting, and T. A. Jung, Chem. Phys. Lett. 411, 214 (2005).

[5] H. Wende, M. Bernien, J. Luo, C. Sorg, N. Ponpandian, J. Kurde, J. Miguel, M. Piantek, X. Xu, P. Eckhold, W. Kuch, K. Baberschke, P. M. Panchmatia, B. Sanyal, P. M. Oppeneer, and O. Eriksson, Nat. Mater. 6, 516 (2007).

[6] M. Bernien, X. Xu, J. Miguel, M. Piantek, P. Eckhold, J. Luo, J. Kurde, W. Kuch, K. Baberschke, H. Wende, and P. Srivastava, Phys. Rev. B 76, 214406 (2007).

[7] M. Bernien, J. Miguel, C. Weis, Md. E. Ali, J. Kurde, B. Krumme, P. M. Panchmatia, B. Sanyal, M. Piantek, P. Srivastava, K. Baberschke, P. M. Oppeneer, O. Eriksson, W. Kuch, and H. Wende, Phys. Rev. Lett. 102, 047202 (2009).

[8] D. Klar, B. Brena, H. C. Herper, S. Bhandary, C. Weis, B. Krumme, C. Schmitz-Antoniak, B. Sanyal, O. Eriksson, and H. Wende, Phys. Rev. B 88, 224424 (2013).

[9] S. Marocchi, P. Ferriani, N. M. Caffrey, F. Manghi, S. Heinze, and V. Bellini, Phys. Rev. B 88, 144407 (2013).

[10] C. F. Hermanns, K. Tarafder, M. Bernien, A. Krüger, Y.-M. Chang, P. M. Oppeneer, and W. Kuch, Adv. Mater. 25, 3473 (2013).

[11] C. F. Hermanns, M. Bernien, A. Krüger, W. Walter, Y.-M. Chang, E. Weschke, and W. Kuch, Phys. Rev. B 88, 104420 (2013).
[12] C. S. Guo, L. Sun, K. Hermann, C. F. Hermanns, M. Bernienand, and W. Kuch, J. Chem. Phys. 137, 194703 (2012).

[13] K. S. Novoselov, A. K. Geim, S. V. Morozov, D. Jiang, Y. Zhang, S. V. Dubonos, I. V. Grigorieva, and A. A. Firsov, Science 306, 666 (2004).

[14] A. K. Geim and K. S. Novoselov, Nat. Mater. 6, 183 (2007).

[15] S. Bhandary, S. Ghosh, H. C. Herper, H. Wende, O. Eriksson, and B. Sanyal, Phys. Rev. Lett. 107, 257202 (2011).

[16] A. Grüneis, K. Kummer, and D. V. Vyalikh, New J. Phys. 11, 073050 (2009).

[17] Y. S. Dedkov and M. Fonin, New J. Phys. 12, 125004 (2010).

[18] See Supplemental Material at http://link.aps.org/supplemental/ 10.1103/PhysRevB.89.144411 for a detailed analysis of the molecules' orientation on the surface and the magnetic field dependence detected by x-ray absorption spectroscopy.

[19] G. Kresse and J. Hafner, Phys. Rev. B 47, R558 (1993); G. Kresse and J. Furthmüller, ibid. 54, 11169 (1996).

[20] V. I. Anisimov, F. Aryasetiawan, and A. I. Lichtestein, J. Phys.: Condens. Matter 9, 767 (1997).

[21] P. M. Panchmatia, B. Sanyal, and P. M. Oppeneer, Chem. Phys. 343, 47 (2008).

[22] S. Grimme, J. Comp. Chem. 27, 1787 (2006).

[23] S. Bhandary et al., Phys. Rev. B 88, 024401 (2013).

[24] S. Bhandary, O. Eriksson, and B. Sanyal, Sci. Rep. 3, 3405 (2013).

[25] X. Chen, Y.-S. Fu, S.-H. Ji, T. Zhang, P. Cheng, X.-C. Ma, X.-L. Zou, W.-H. Duan, J.-F. Jia, and Q.-K. Xue, Phys. Rev. Lett. 101, 197208 (2008). 